\section{Dependência de álcool e fatores associados em adultos residentes em Ribeirão Preto, São Paulo, Brasil, 2006: Projeto OBEDIARP}

\author{
Alcohol addiction and associated factors in adults \\ in Ribeirão Preto, São Paulo State, Brazil, 2006: \\ the OBEDIARP Project
}

\author{
${ }_{1}$ Escola de Enfermagem de \\ Ribeirão Preto, Universidade \\ de São Paulo, Ribeirão Preto, \\ Brasil. \\ Correspondência \\ S. A. Moraes \\ Escola de Enfermagem de \\ Ribeirão Preto, Universidade \\ de São Paulo. \\ Av. Bandeirantes 3900, \\ Ribeirão Preto, SP \\ 14040-902, Brasil. \\ samoraes@usp.br
}

\begin{abstract}
This study aims to estimate the prevalence and severity of alcohol addiction and to identify associated factors. A cross-sectional populationbased study was conducted in Ribeirão Preto, São Paulo State, Brazil, in 2006. Prevalence estimates were based on AUDIT (Alcohol Use Disorders Identification Test). Crude and adjusted prevalence ratios $(P R)$ were calculated, and the outcome variable was classified in two levels: (1) scores $=0-7$; and (2) scores $=8-40$. Overall prevalence of alcohol addiction was $20.18 \%$ (43.47\% in men and $10.18 \%$ in women). In the final model, the following variables were associated with alcohol addiction: gender, age, schooling, marital and work status, smoking, religion, and self-rated health. Prevalence of alcohol addiction was high, especially among men. The results should contribute to the adoption of health promotion and prevention strategies, considering the consequences of alcohol addiction.
\end{abstract}

Alcoholism; Cross-Sectional Studies; Questionnaires; Prevalence
Isabel Cristina Martins de Freitas 1 Suzana Alves de Moraes 1

\section{Introdução}

Com base na literatura disponível nas últimas décadas, o consumo moderado de bebidas alcoólicas pode trazer, em geral, algum benefício para a saúde das pessoas e contribuir para a redução da mortalidade por doenças cardiovasculares. Por outro lado, o consumo abusivo tem sido responsável por grande número de acidentes de trânsito e de trabalho, violência doméstica e aumento da morbi-mortalidade por doenças cardiovasculares, cirrose hepática, acidentes vasculares cerebrais e distúrbios psiquiátricos 1 .

Grucza et al. 2, ao analisarem dois estudos transversais conduzidos nos Estados Unidos, em 1991/ 1992 e 2001/2002, utilizando o DSM-IV (Diagnostic and Statistical Manual of Disorders $4^{\text {th }}$ version) para avaliar o padrão de consumo de bebidas alcoólicas e dependência de álcool, encontraram prevalências, ajustadas para coortes de nascimento, em torno de $17 \%$ para o consumo e de $20 \%$ para a dependência. Nos dois estudos, os autores observaram prevalência de dependência de maior magnitude no sexo masculino. Resultados de outros dois estudos transversais conduzidos por Giesbrecht et al. 3 em Ontário (Canadá), após aplicação do AUDIT (Alcohol Use Disorder Identification Test), revelaram prevalência de dependência de álcool de 13\%, sendo maior em participantes do sexo masculino.

Em estudo ecológico conduzido em países das Américas, no ano 2000, pela Organização 
Mundial da Saúde (OMS) ${ }^{4}$, investigou-se a relação entre o padrão de consumo de álcool e as taxas de mortalidade, juntamente com anos de vida perdidos por incapacidades (Disability Adjusted Life Years - DALYs). Os resultados indicaram que a média de consumo de álcool foi $50 \%$ maior que no resto do mundo, correspondendo a 5\% dos óbitos e 9,7\% de DALYs. O Brasil, classificado na região $\mathrm{B}$ (baixas taxas de mortalidade infantil e geral), apresentou média de consumo anual per capita de cerca de 11 litros. No sexo feminino, o consumo abusivo ( $\geq 20 \mathrm{~g}$ ) correspondeu a 3,5\% dos óbitos e 4,1\% de DALYs, enquanto no masculino (consumo abusivo $\geq 40 \mathrm{~g}$ ) os respectivos indicadores foram $14,2 \%$ e $17,3 \%$. Os autores concluíram ainda que, para o conjunto dos países pertencentes à região $\mathrm{B}$, o consumo abusivo de álcool foi considerado o fator de risco mais importante para a carga de doenças que resultou em $11,4 \%$ de anos de vida perdidos por incapacidades.

Em artigo de atualização na área de Economia em Saúde, Moraes et al. 5 avaliaram o impacto social do consumo abusivo de álcool no Brasil, de 1995-1997, e apontaram que em $61 \%$ dos acidentes de trânsito, ocorridos no período, os condutores apresentaram algum grau de alcoolemia, enquanto o gasto estimado com internações, decorrentes da dependência foi de, aproximadamente, $\mathrm{R} \$ 310$ milhões.

Entre os estudos transversais conduzidos no Brasil em população adulta, Almeida-Filho et al. 6 detectaram que a prevalência de consumo de alto risco ( $\geq 2$ doses/dia) em Salvador, Bahia, foi de $6,9 \%$, sendo $2,3 \%$ e $12,4 \%$ para os sexos masculino e feminino, respectivamente. Galduróz et al. 7, em pesquisa realizada em 24 cidades do Estado de São Paulo em 1999, relataram prevalência de $55,6 \%$ para o consumo habitual de álcool em indivíduos com 35 anos ou mais. A dependência de álcool, avaliada usando-se o DSM-III (Diagnostic and Statistical Manual of Disorders - 3rd version), foi da ordem de $5 \%$, sendo $8,9 \%$ para os homens e $1,3 \%$ para as mulheres, classificados nessa faixa etária.

Fatores associados ao consumo habitual de álcool ( $\geq 4$ vezes/semana) foram investigados em estudo transversal de abrangência nacional, conduzido em 2005 por Bastos et al. 8, com participantes de 16-65 anos de idade. Permaneceram no modelo final as variáveis: idade; ter vivido, durante a infância, em um lar que não considerava a religião importante; e ser "não branco".

Resultados de estudo transversal, vinculado ao Multi-center Intervention Study of Suicide Behavior da OMS, em 2003, indicaram para o Município de Campinas, São Paulo, Brasil 9, prevalências de dependência de álcool de 13,1\% para o sexo masculino e de $4,1 \%$ para o feminino, após a aplicação do questionário AUDIT. Nesse estudo, permaneceram associadas à dependência as variáveis: idade, renda, escolaridade, não ter religião e uso de drogas ilícitas.

No que pese a relevância do consumo abusivo de álcool e suas consequências para a saúde, grande parte das pesquisas conduzidas no Brasil restringe-se a resultados descritivos, apresentados para o país como um todo, e que nem sempre correspondem à realidade específica de suas macrorregiões ou de seus municípios. Por outro lado, instrumentos não validados têm sido aplicados, o que dificulta a comparação de resultados.

Diante do panorama apresentado, o presente estudo, desenvolvido em base populacional, teve por objetivo estimar a prevalência da dependência de álcool e a gravidade do hábito em população adulta, residente na área urbana do Município de Ribeirão Preto, buscando-se identificar os fatores associados à dependência.

\section{Material e métodos}

\section{Delineamento do estudo e processo de amostragem}

O presente trabalho está vinculado ao Projeto OBEDIARP 10, conduzido no Município de Ribeirão Preto em 2006, com delineamento transversal e seleção de participantes realizada em três estágios. Foram sorteados 81 setores censitários (Instituto Brasileiro de Geografia e Estatística. Censo Demográfico 2000. http://www.ibge.gov.br), 1.671 endereços e 1.205 participantes nos primeiro, segundo e terceiro estágios, respectivamente. O método de sorteio por conglomerados, sob partilha proporcional ao tamanho, foi adotado nos dois primeiros estágios. No segundo, foi introduzida a estratificação por renda nominal média do chefe da família. No terceiro estágio, sorteou-se uma pessoa com 30 anos ou mais entre os $(\mathrm{N})$ residentes nos domicílios sorteados, levando-se também em consideração a estratificação por faixa etária, em ambos os sexos 11 . O setor censitário foi considerado a unidade primária de amostragem, e a amostra de participantes foi composta por 930 adultos com 30 anos e mais de idade, residentes no município. A taxa de resposta do Projeto OBEDIARP foi de $78 \%$, sendo as perdas distribuídas da seguinte forma: $6,6 \%$ por mudanças, $0,6 \%$ por óbitos e $14,6 \%$ por recusas, após seis tentativas de contato em dias, períodos e horários alternados. Mulheres gestantes e puérperas até o sexto mês foram excluídas do estudo $(0,7 \%)$. 
A variabilidade introduzida nas segunda e terceira frações de amostragem foi levada em consideração, atribuindo-se pesos amostrais 12 para a correção da taxa de não-resposta e número de elegíveis em cada domicílio, originando, assim, uma amostra ponderada de 2.197 participantes. O detalhamento sobre as técnicas utilizadas para ponderação da amostra encontra-se em Moraes et al. 10. A média de entrevistas por setor censitário foi de 11,5.

\section{Processamento dos dados}

A coleta das informações foi realizada por meio da aplicação de um questionário estruturado, mediante entrevistas domiciliares, realizadas por 10 duplas de entrevistadores, previamente treinados. A equipe de entrevistadores foi constituída por profissionais de níveis médio e superior, com experiência comprovada em entrevistas domiciliares. As entrevistas foram previamente agendadas e conduzidas diariamente, inclusive nos finais de semana. Antes da coleta de dados propriamente dita, conduziu-se um estudo piloto que incluiu $10 \%$ do total de entrevistas $(\mathrm{n}=$ 100), realizadas em diferentes setores censitários. Nessa ocasião, alguns ajustes no questionário foram efetuados com o propósito de facilitar o entendimento das questões, por parte dos entrevistados.

Para a entrada de dados utilizou-se o aplicativo EPIDATA, versão 2.1 (Epidata Association, Odense, Dinamarca) e as informações foram digitadas sob a forma de "dupla entrada" para a análise de consistência externa.

O controle de qualidade das informações foi avaliado por meio de replicação de $12,5 \%$ das entrevistas. A reprodutibilidade obtida utilizandose a estatística kappa foi superior a 0,80 em todas as questões incluídas na replicação (informações não passíveis de mudança no tempo).

\section{Variáveis do estudo}

\section{- Variável dependente}

A dependência de álcool e a gravidade do hábito foram avaliadas mediante a aplicação do questionário AUDIT, desenvolvido pela OMS 13 e validado no Brasil por Lima et al. 14. Os escores do AUDIT podem variar de 0-40, e referem-se ao hábito de consumir bebidas alcoólicas, tendo como referência, o último ano que antecedeu a entrevista. Para o cálculo das prevalências, esta variável foi inicialmente classificada em duas categorias: sem dependência (escores de 0-7) e com algum grau de dependência (escores de 8-40). Posteriormente, utilizou-se a classificação em quatro níveis indicativos da gravidade do hábito, também denominados zonas de "risco": zona I (escores de 0-7), zona II (de 8-15), zona III (de 16-19) e zona IV (escores de 20-40).

Na fase analítica, a variável dependente foi classificada de forma dicotômica, utilizando-se como ponto de corte o escore 815 e considerando-se os escores de 0-7 como categoria de referência.

\section{- Variáveis independentes}

As variáveis independentes foram agrupadas em três blocos:

i) Bloco 1 - variáveis socioeconômicas e demográficas: sexo; idade (calculada a partir das diferenças entre a data da entrevista e a data de nascimento, dividida por 365,25 , e, posteriormente, classificada em intervalos de 10 anos); escolaridade (obtida com base no número de anos completos de escolaridade formal e, posteriormente, classificada em 4 categorias: 0 -3; 4-7; 8-10 e 11 anos ou mais); estado marital (com ou sem companheiro(a), no momento da entrevista, independentemente de união formal); condição de trabalho (sim/não, tendo como período de referência o mês que antecedeu a entrevista); e renda individual (valores nominais da renda, declarada em Reais, no mês que antecedeu a entrevista e que foram tratados sob a forma de tercis, sendo que os participantes que declararam não ter trabalho, no mês que antecedeu a entrevista, foram classificados como sem renda).

ii) Bloco 2 - variáveis comportamentais: hábito de fumar (não fumantes, ex-fumantes e fumantes); religião e sua importância (cada participante declarou sua filiação religiosa e qualificou o grau de importância desta filiação em suas vidas: importante, regular ou nada importante).

iii) Bloco 3 - estado de saúde: autopercepção do estado de saúde, no momento da entrevista, classificado em duas categorias: excelente/bom ou regular/ruim/péssimo.

\section{Análise estatística dos dados}

As distribuições de frequências absolutas e relativas das variáveis do estudo foram calculadas por pontos e por intervalos com 95\% de confiança (IC95\%), estratificadas segundo o sexo dos participantes (caracterização da amostra).

A prevalência da dependência de álcool foi estimada segundo o sexo dos participantes. Prevalências correspondentes aos níveis de gravidade do hábito (zonas de risco) foram calculadas levando-se em consideração as variáveis socioeconômicas e demográficas, comportamentais e a autoavaliação do estado de saúde. Para a de- 
tecção de associações globais entre as variáveis independentes e os níveis de gravidade (fase descritiva), utilizou-se a estatística F com correção para efeito de desenho amostral.

Na fase analítica, para a investigação de associações entre as variáveis independentes e a variável resposta (dependência de álcool), razões de prevalências 16 foram estimadas por pontos e por IC95\%, por meio da regressão de Poisson 17. Modelos univariados foram construídos e, a seguir, optou-se por considerar a ordem decrescente, em magnitude, da estatística F como critério de entrada de variáveis para a construção dos modelos multivariados. No modelo final, permaneceram as variáveis que, após o ajustamento simultâneo, apresentaram valores $\mathrm{p}<0,05$ para o teste de Wald. As variáveis independentes, classificadas em mais de duas categorias, foram tratadas sob a forma de variáveis indicadoras (dummy), aplicando-se testes de tendência linear para as razões de prevalências, desde que as estimativas indicassem gradiente linear.

Todas as análises foram processadas no módulo survey do aplicativo Stata, versão 8.2 (Stata Corp., College Station, Estados Unidos), e todas as estimativas levaram em consideração o efeito de desenho amostral 12. A utilização desse módulo específico permite o cálculo de variâncias em amostras complexas (múltiplos estágios), considerando-se a probabilidade desigual de sorteio nas diferentes etapas do processo de amostragem.

\section{Considerações éticas}

O Projeto OBEDIARP foi submetido e aprovado em junho de 2005 pelo Comitê de Ética em Pesquisa da Escola de Enfermagem de Ribeirão Preto, Universidade de São Paulo (CEP-EERP/USP), e foi protocolado sob o no. $0528 / 2005$. Todos os participantes assinaram o Termo de Consentimento Livre e Esclarecido.

\section{Resultados}

Na Tabela 1, apresenta-se a caracterização da amostra do estudo segundo variáveis socioeconômicas e demográficas, comportamentais e autoavaliação do estado de saúde, segundo o sexo dos participantes. De modo geral, as características apontadas foram semelhantes em ambos os sexos. Vale destacar que mais da metade dos participantes do sexo feminino possuía trabalho no mês que antecedeu a entrevista, e somente $15,82 \%$ foram classificados no terceiro terço de renda. Quanto ao hábito de fumar, 61,83\% das mulheres eram fumantes. Menos de 5\% declara- ram não pertencer a nenhuma religião e 93,86\% classificaram sua filiação religiosa como muito importante para suas vidas. Mais de um terço considerou seu estado de saúde ruim/péssimo.

A prevalência de dependência de álcool (Tabela 2) foi de $20,18 \%$ (efeito de desenho amostral $=1,1497$ ), sendo $43,47 \%$ e $10,18 \%$ para os sexos masculino e feminino, respectivamente.

A Tabela 3 exibe a classificação dos participantes em níveis de gravidade do hábito de consumir bebida alcoólica (zonas de risco), segundo as variáveis do estudo. Cerca de $15 \%$ dos participantes do sexo masculino foram classificados nos níveis III e IV e, aproximadamente, 30\% dos mais jovens (30-39 anos) já se encontravam nos níveis II, III e IV. Quase 6\% dos que viviam sem companheiro(a) ou daqueles classificados no terceiro terço de renda e cerca de $10 \%$ dos fumantes, concentraram-se no nível III. Foram classificados no nível IV, cerca de $8 \%$ do sexo masculino ou dos que não tinham nenhuma religião, e quase 5\% dos que consideraram seu estado de saúde como regular/ruim/péssimo.

Razões de prevalências brutas e ajustadas, com respectivos IC95\%, são apresentadas na Tabela 4 . As variáveis que permaneceram no modelo final foram: sexo, faixa etária, nível educacional, estado marital, condição de trabalho, hábito de fumar, religião e autopercepção do estado de saúde. A razão de prevalências foi, aproximadamente, três vezes maior no sexo masculino, quando comparada ao sexo feminino e, entre os mais jovens, (30-39 anos), foi duas vezes maior quando comparada aos mais velhos (60 anos e mais). As razões de prevalências foram mais elevadas naqueles que tinham 4-7 anos de escolaridade; nos que viviam sem companheiro(a); nos que estavam trabalhando no momento da entrevista; e em "fumantes" e "ex-fumantes", quando comparados com suas respectivas categorias de referência. Quanto à filiação religiosa, observouse razão de prevalências de menor magnitude entre "Protestantes" quando comparados com aqueles que declararam "não ter religião".

Tendência linear ( $p<0,001)$ foi identificada, nos modelos uni e multivariados, para as razões de prevalências nas categorias da variável "hábito de fumar".

\section{Discussão}

Os resultados do presente estudo apontaram elevada prevalência de dependência de álcool na população e permitiram a identificação de fatores associados ao desfecho de interesse, entre os quais destacaram-se as variáveis sociodemográficas, filiação religiosa e autopercepção do estado 
Características da população do estudo, segundo sexo. Projeto OBEDIARP, Ribeirão Preto, São Paulo, Brasil, 2006.

\begin{tabular}{|c|c|c|c|c|c|}
\hline & \multirow[t]{2}{*}{$\mathbf{N}_{\mathrm{w}}$ * } & \multicolumn{2}{|c|}{ Masculino $\left(\mathrm{N}_{\mathrm{w}}{ }^{*}=660,1\right)$} & \multicolumn{2}{|c|}{ Feminino $\left(N_{w}{ }^{*}=1537,0\right)$} \\
\hline & & $\%$ * & IC $95 \%$ * & $\%$ * & IC95\% * \\
\hline \multicolumn{6}{|l|}{ Faixas etárias (anos) } \\
\hline$\geq 60$ & 420,3 & 18,72 & $14,13-24,37$ & 19,31 & $15,88-23,27$ \\
\hline $50-59$ & 454,3 & 19,04 & $14,93-23,97$ & 21,38 & $17,87-25,37$ \\
\hline $40-49$ & 667,0 & 24,24 & $20,00-29,05$ & 32,98 & $29,61-36,54$ \\
\hline $30-39$ & 655,4 & 38,00 & $32,29-44,06$ & 26,33 & $22,16-30,97$ \\
\hline \multicolumn{6}{|l|}{ Nível educacional (anos) } \\
\hline$\geq 11$ & 853,6 & 41,28 & $34,32-48,62$ & 37,81 & $32,48-43,45$ \\
\hline $8-10$ & 320,9 & 13,28 & $9,79-17,76$ & 15,18 & $12,33-18,54$ \\
\hline $4-7$ & 679,3 & 31,29 & $25,70-37,49$ & 30,76 & $26,65-35,21$ \\
\hline $0-3$ & 343,2 & 14,15 & $10,58-18,66$ & 16,25 & $13,32-19,68$ \\
\hline \multicolumn{6}{|l|}{ Estado marital } \\
\hline Com companheiro(a) & $1.617,0$ & 75,96 & $70,06-81,01$ & 72,56 & $68,81-76,03$ \\
\hline Sem companheiro(a) & 580,4 & 24,04 & $18,99-29,94$ & 27,44 & $23,97-31,19$ \\
\hline \multicolumn{6}{|l|}{ Condição de trabalho } \\
\hline Não & 825,5 & 20,18 & $15,99-25,14$ & 45,05 & $40,50-49,68$ \\
\hline Sim & $1.371,5$ & 79,82 & $74,86-84,01$ & 54,95 & $50,32-59,50$ \\
\hline \multicolumn{6}{|l|}{ Renda individual (em $\mathrm{R} \$$ ) } \\
\hline Nenhuma & 825,5 & 20,18 & $15,99-25,14$ & 45,05 & $40,50-49,68$ \\
\hline 1ㅇ terço $(60,00-520,00)$ & 438,1 & 9,52 & $5,84-15,13$ & 24,41 & $20,37-28,96$ \\
\hline 2o terço $(520,01-1.100,00)$ & 442,3 & 32,73 & $26,52-39,60$ & 14,72 & $12,29-17,55$ \\
\hline 3o terço $(>1.100,00)$ & 491,1 & 37,58 & $31,08-44,55$ & 15,82 & $12,18-20,28$ \\
\hline \multicolumn{6}{|l|}{ Hábito de fumar } \\
\hline Nunca fumou & $1.211,0$ & 24,51 & $19,11-30,85$ & 18,73 & $16,30-21,43$ \\
\hline Ex-fumante & 536,6 & 36,06 & $30,61-41,90$ & 19,44 & $16,47-22,79$ \\
\hline Fumante & 449,4 & 39,43 & $33,69-45,49$ & 61,83 & $57,57-65,92$ \\
\hline \multicolumn{6}{|l|}{ Religião } \\
\hline Nenhuma & 132,8 & 10,17 & $6,54-15,49$ & 4,27 & $2,83-6,39$ \\
\hline Protestante & 432,5 & 16,29 & $11,70-22,22$ & 21,15 & $17,42-25,42$ \\
\hline Católica & $1.436,2$ & 66,47 & $59,90-72,47$ & 64,91 & $60,61-68,98$ \\
\hline Outras & 195,5 & 7,07 & $4,61-10,69$ & 9,67 & $7,29-12,73$ \\
\hline \multicolumn{6}{|l|}{ Importância da religião } \\
\hline Muito importante & $1.870,0$ & 82,53 & $76,48-87,29$ & 93,86 & $91,15-95,78$ \\
\hline Pouco/Nada importante & 193,9 & 17,47 & $12,71-23,52$ & 6,14 & $4,22-8,85$ \\
\hline \multicolumn{6}{|c|}{ Estado de saúde (autopercepção) } \\
\hline Excelente/Bom & $1.477,2$ & 78,17 & $73,59-82,15$ & 62,54 & $58,48-66,44$ \\
\hline Regular/Ruim/Péssimo & 719,8 & 21,83 & $17,85-26,41$ & 37,46 & $33,56-41,52$ \\
\hline
\end{tabular}

* Estimativas ponderadas, considerando-se o efeito de desenho amostral.

de saúde. Ao lado do rigor metodológico com que foram conduzidas todas as etapas do trabalho, desde o processo de amostragem até o controle de qualidade das informações, treinamento dos entrevistadores e elevada taxa de resposta, destaca-se a composição de amostra representativa de adultos residentes no Município de Ribeirão Preto, que, em conjunto, contribuíram para mi- nimizar vícios de seleção e aferição, reforçando a validade interna do estudo.

O questionário AUDIT tem apresentado bom desempenho em estudos clínicos e observacionais de base populacional 18. Estudos de validação 18,19,20 que compararam os escores do AUDIT com marcadores biológicos de dependência de álcool (gama-glutamiltransferase e volume 
Prevalência de dependência de álcool, segundo sexo. Projeto OBEDIARP, Ribeirão Preto, São Paulo, Brasil, 2006.

\begin{tabular}{lcccccc}
\hline \multirow{2}{*}{ AUDIT (escores) } & \multicolumn{2}{c}{ Masculino } & \multicolumn{2}{c}{ Feminino } & \multicolumn{2}{c}{ Total } \\
& $\mathbf{N}_{\mathbf{w}}^{*}$ & $\% *$ & $\mathbf{N}_{\mathbf{w}}^{*}$ & $\% *$ & $\mathbf{N}_{\mathbf{w}}^{*}$ & \\
\hline $0-7$ & 373,0 & 56,53 & $1.380,5$ & 89,82 & $1.754,0$ & 79,82 \\
$8-40$ & 287,0 & 43,47 & 156,5 & 10,18 & 443,0 & 20,18 \\
Total & 660,0 & 100,00 & $1.537,0$ & 100,00 & $2.197,0$ & 100,00 \\
\hline
\end{tabular}

AUDIT: Alcohol Use Disorder Identification Test.

* Estimativas ponderadas, considerando-se o efeito de desenho amostral.

corpuscular médio) apresentaram sensibilidade e especificidade superiores a 0,95 e acurácia global equivalente a 0,92 , enquanto os resultados do COMBINE Study 20 revelaram que as zonas de risco do AUDIT reproduziram os níveis de gravidade do DSM-IV.

Pesquisas brasileiras conduzidas em diferentes localidades 6,21,22 que avaliaram a dependência de álcool em população adulta, apontaram prevalências de maior magnitude para o sexo masculino. No entanto, as estimativas brutas ou estratificadas por sexo foram menores, quando comparadas às do presente estudo. Essas diferenças podem ser decorrentes dos intervalos de idade das populações pesquisadas, do instrumento utilizado para avaliar a dependência e, principalmente, da definição usada para a sua classificação, que, naqueles estudos, foi considerada equivalente a consumo abusivo episódico. Vale destacar que consumidores habituais, que ainda não apresentaram padrão que inclua episódios de consumo abusivo, já podem ter adquirido algum grau de dependência de álcool o que explicaria, em parte, prevalência de maior magnitude na população adulta de Ribeirão Preto.

No que pesem as diferenças metodológicas, principalmente relacionadas à aplicação de diferentes instrumentos para aferição da dependência de álcool, os resultados da fase analítica do presente estudo aproximaram-se daqueles encontrados por outros autores 9,21,22,23,24,25, destacando-se, entretanto, o achado de razões de prevalências de elevada magnitude nos participantes mais jovens, do sexo masculino e nos fumantes, que quase triplicaram em relação às respectivas categorias de referência.

Laranjeira et al. 24, em estudo transversal conduzido com amostra representativa da população brasileira de 18 anos e mais, em 2006, avaliaram o padrão de consumo de álcool por meio da aplicação de questionário adaptado e submetido à validação transcultural do Instrumento utilizado no
HABLAS - Hispanic Americans Baseline Alcohol Survey. Na fase descritiva do trabalho brasileiro, o consumo abusivo de álcool em uma única ocasião ( $\geq 5$ doses para homens $\mathrm{e} \geq 4$ doses para mulheres), tendo como referência os 12 meses que antecederam a entrevista, apresentou prevalência bruta de $28 \%$, sendo $40 \%$ no sexo masculino e $18 \%$ no feminino. Na fase analítica, os autores identificaram o sexo, a idade, o estado marital, a escolaridade e a renda familiar como fatores associados à dependência de álcool, após ajustamento simultâneo, que também incluiu variáveis de nível agregado como macrorregiões, área e local de residência. Esses resultados, em suas fases descritiva e analítica, foram semelhantes aos do presente estudo, ainda que relacionados a populações mais jovens e de diferentes regiões do país.

Relações entre gene e ambiente estão no centro do debate sobre a origem de várias doenças e também do alcoolismo. A dependência química e os distúrbios mentais são doenças decorrentes da atuação de múltiplos fatores, além da interação gene/ambiente. Por outro lado, a herança genética do alcoolismo é também compartilhada com a do tabagismo, o que pode explicar a forte associação encontrada, na maioria dos estudos, entre estes dois hábitos 26 . A associação independente entre o hábito de fumar e a dependência de álcool encontrada no presente estudo, bem como a detecção de gradiente linear para as razões de prevalências do desfecho (dependência de álcool) nas categorias da variável "hábito de fumar”, reforçam a hipótese de uma origem comum entre estes hábitos.

A filiação religiosa tem sido considerada como fator associado à dependência de álcool, conforme resultados relatados por Bastos et al. 8 e Barros et al. ${ }^{9}$. Esses autores sugerem que a prática religiosa pode exercer efeito protetor sobre comportamentos de risco de dependência, principalmente quando relacionada a cultos que 


\section{Tabela 3}

Níveis de gravidade * (\%) do hábito, segundo características da população do estudo. Projeto OBEDIARP, Ribeirão Preto, São Paulo, Brasil, 2006.

\begin{tabular}{|c|c|c|c|c|}
\hline & \multicolumn{4}{|c|}{ Zonas de "risco" - AUDIT } \\
\hline & $I(0-7)$ ** & II $(8-15)$ ** & III $(16-19) * *$ & IV $(20-40)$ ** \\
\hline \multicolumn{5}{|l|}{ Sexo *** } \\
\hline Feminino & 89,81 & 7,70 & 1,28 & 1,21 \\
\hline Masculino & 56,53 & 28,68 & 7,24 & 7,55 \\
\hline \multicolumn{5}{|l|}{ 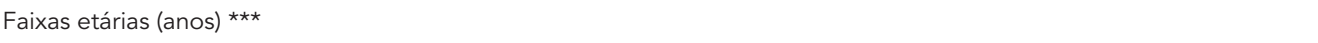 } \\
\hline$\geq 60$ & 89,53 & 9,45 & 0,50 & 0,52 \\
\hline $50-59$ & 79,97 & 11,61 & 4,50 & 3,92 \\
\hline $40-49$ & 82,28 & 12,40 & 2,59 & 2,73 \\
\hline $30-39$ & 70,98 & 20,20 & 4,22 & 4,60 \\
\hline \multicolumn{5}{|l|}{ Nível educacional (anos) } \\
\hline$\geq 11$ & 82,56 & 13,09 & 2,85 & 1,50 \\
\hline $8-10$ & 78,96 & 13,71 & 3,70 & 3,63 \\
\hline $4-7$ & 75,20 & 16,91 & 3,34 & 4,55 \\
\hline $0-3$ & 82,93 & 10,76 & 2,52 & 3,79 \\
\hline \multicolumn{5}{|l|}{ 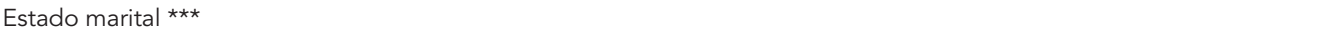 } \\
\hline Com companheiro(a) & 82,07 & 13,55 & 2,02 & 2,36 \\
\hline Sem companheiro(a) & 73,54 & 15,26 & 6,00 & 5,20 \\
\hline \multicolumn{5}{|l|}{ 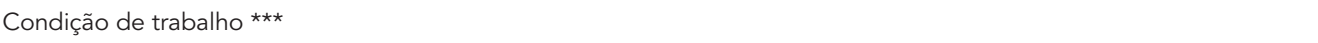 } \\
\hline Não & 88,59 & 7,84 & 1,44 & 2,13 \\
\hline Sim & 74,54 & 17,70 & 4,06 & 3,70 \\
\hline \multicolumn{5}{|l|}{ 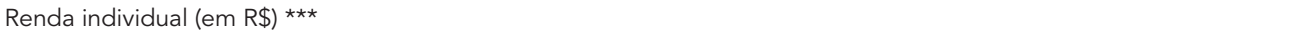 } \\
\hline Nenhuma & 88,59 & 7,84 & 1,44 & 2,13 \\
\hline 1ㅇ terço $(60,00-520,00)$ & 81,22 & 12,97 & 2,01 & 3,80 \\
\hline 2o terço $(520,01-1.100,00)$ & 70,65 & 20,61 & 3,48 & 5,26 \\
\hline 3o terço $(>1.100,00)$ & 72,07 & 19,30 & 6,41 & 2,22 \\
\hline \multicolumn{5}{|l|}{ 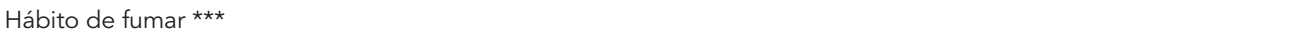 } \\
\hline Nunca fumou & 89,91 & 8,05 & 0,72 & 1,32 \\
\hline Ex-fumante & 76,93 & 16,45 & 2,51 & 4,11 \\
\hline Fumante & 56,08 & 27,10 & 10,08 & 6,74 \\
\hline \multicolumn{5}{|l|}{ 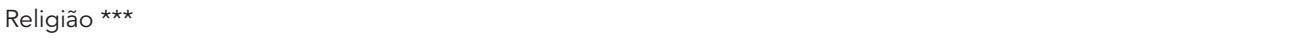 } \\
\hline Nenhuma & 66,90 & 19,04 & 5,70 & 8,36 \\
\hline Protestante & 93,38 & 3,81 & 1,67 & 1,14 \\
\hline Católica & 75,85 & 17,78 & 2,85 & 3,52 \\
\hline Outras & 87,71 & 5,32 & 6,00 & 0,97 \\
\hline \multicolumn{5}{|l|}{ Importância da religião *** } \\
\hline Muito importante & 81,90 & 12,66 & 2,83 & 2,61 \\
\hline Pouco ou nada importante & 68,56 & 23,44 & 3,62 & 4,38 \\
\hline \multicolumn{5}{|c|}{ Estado de saúde (autopercepção) } \\
\hline Excelente/Bom & 80,14 & 14,28 & 3,11 & 2,47 \\
\hline Regular/Ruim/Péssimo & 79,16 & 13,41 & 3,00 & 4,43 \\
\hline
\end{tabular}

AUDIT: Alcohol Use Disorder Identification Test.

* Estimativas ponderadas, considerando-se o efeito de desenho amostral;

** Pontos de corte utilizados para a classificação das zonas de "risco";

*** Valores de $p<0,05$ para a estatística F. 
Razões de prevalências * (RP) brutas e ajustadas para a dependência de álcool (modelo final). Projeto OBEDIARP, Ribeirão Preto, São Paulo, Brasil, 2006.

\begin{tabular}{|c|c|c|c|c|}
\hline & RP brutas & IC95\% & RP ajustadas & IC95\% \\
\hline \multicolumn{5}{|l|}{ Sexo } \\
\hline Feminino & 1,00 & - & 1,00 & - \\
\hline Masculino & 4,26 & $3,23-5,63$ & 3,32 & $2,48-4,45$ \\
\hline \multicolumn{5}{|l|}{ Faixas etárias (anos) } \\
\hline$\geq 60$ & 1,00 & - & 1,00 & - \\
\hline $50-59$ & 1,91 & $1,06-3,45$ & 1,59 & $0,99-2,55$ \\
\hline $40-49$ & 1,69 & $1,02-2,78$ & 1,55 & $0,97-2,46$ \\
\hline $30-39$ & 2,77 & $1,67-4,59$ & 2,48 & $1,53-4,00$ \\
\hline \multicolumn{5}{|l|}{ Nível educacional (anos) } \\
\hline$\geq 11$ & 1,00 & - & 1,00 & - \\
\hline $8-10$ & 1,20 & $0,78-1,84$ & 1,21 & $0,86-1,69$ \\
\hline $4-7$ & 1,42 & $1,10-1,82$ & 1,33 & $1,05-1,68$ \\
\hline $0-3$ & 0,97 & $0,66-1,44$ & 1,10 & $0,76-1,58$ \\
\hline \multicolumn{5}{|l|}{ Estado marital } \\
\hline Com companheiro(a) & 1,00 & - & 1,00 & - \\
\hline Sem companheiro(a) & 1,47 & $1,11-1,96$ & 1,45 & $1,18-1,77$ \\
\hline \multicolumn{5}{|l|}{ Condição de trabalho } \\
\hline Não & 1,00 & - & 1,00 & - \\
\hline Sim & 2,23 & $1,61-3,08$ & 1,40 & $1,08-1,81$ \\
\hline \multicolumn{5}{|l|}{ Hábito de fumar ** } \\
\hline Nunca fumou & 1,00 & - & 1,00 & - \\
\hline Ex-fumante & 2,28 & $1,55-3,36$ & 1,86 & $1,31-2,64$ \\
\hline Fumante & 4,35 & $3,15-6,00$ & 2,75 & $1,95-3,88$ \\
\hline \multicolumn{5}{|l|}{ Religião } \\
\hline Nenhuma & 1,00 & - & 1,00 & - \\
\hline Protestante & 0,20 & $0,10-0,39$ & 0,37 & $0,21-0,64$ \\
\hline Católica & 0,73 & $0,47-1,14$ & 1,10 & $0,76-1,57$ \\
\hline Outras & 0,37 & $0,18-0,76$ & 0,59 & $0,31-1,13$ \\
\hline \multicolumn{5}{|c|}{ Estado de saúde (autopercepção) } \\
\hline Excelente/Bom & 1,00 & - & 1,00 & - \\
\hline Regular/Ruim/Péssimo & 1,04 & $0,80-1,35$ & 1,33 & $1,05-1,69$ \\
\hline
\end{tabular}

IC95\%: intervalo de 95\% de confiança; RP: razão de prevalência.

* Estimativas ponderadas, considerando-se o efeito de desenho amostral;

** Valor de $p<0,001$ para o teste de tendência linear (RP brutas e ajustadas).

impõem normas rígidas contra o hábito, ao lado do oferecimento de uma rede de suporte social que desencoraja o consumo de qualquer quantidade de bebida alcoólica. Os resultados do presente estudo parecem confirmar essas hipóteses ao detectarem que a filiação a cultos protestantes apresentou razão de prevalências menor que a unidade $(R P=0,37)$, indicando que tal filiação confere $63 \%$ de proteção em relação à categoria de referência (nenhuma filiação religiosa).

No presente estudo, a investigação da prevalência de consumo de álcool, segundo zonas de risco, parece pertinente tendo em vista os achados de Dawson et al. 27, que entre duas visitas de seguimento de uma coorte, conduzida nos Estados Unidos (2001-2004), relataram que a redução do consumo de álcool entre as duas visitas somente pode ser verificada naqueles classificados como consumidores sem distúrbios relacionados ao álcool. Os consumidores abusivos ou dependentes apresentaram, em geral, mudanças negativas (aumento do consumo e da tolerância, em relação ao número de doses) após três anos de seguimento. Esses resultados indicaram ainda 
que, mesmo em curtos períodos de observação, o agravamento do consumo de álcool entre consumidores abusivos e dependentes já pode ser detectado, e projeções ainda mais negativas podem ser esperadas a médio e longo prazo, se intervenções precoces na fase de consumo moderado não forem capazes de interceptar seu percurso, antes que mudanças neurobiológicas irreversíveis estejam instaladas.

No que pesem as possíveis diferenças na prevalência da dependência do álcool em diferentes regiões do Brasil, os achados do presente estudo revelaram elevada prevalência deste distúrbio no Município de Ribeirão Preto e, sobretudo, confirmaram um elenco de fatores associados a este desfecho, alguns dos quais passíveis de intervenção. Além da elevada prevalência, há que se considerar os achados de Peluso \& Blay ${ }^{28}$, que em estudo transversal conduzido em São Paulo em 2002, com participantes de 18-65 anos, detectaram que somente $18,8 \%$ dos entrevistados identificaram o alcoolismo como distúrbio mental, apontando o desemprego, uso de drogas ilícitas, comportamento antissocial, problemas familiares, problemas de caráter e falta de fé em Deus como as principais causas de dependência.

Embora o questionário AUDIT tenha sido recomendado pela OMS para a identificação da prevalência de dependência de consumo de álcool e seus níveis de gravidade em estudos epidemiológicos, poucas pesquisas no Brasil têm utilizado este Instrumento para este fim, o que dificulta a comparação com os resultados de trabalhos conduzidos no território nacional. Se, por um lado, os estudos transversais estão sujeitos ao viés de causalidade reversa, os resultados do presente trabalho, principalmente em sua fase analítica, muito se assemelham aos que foram relatados na literatura internacional que utilizaram desenhos prospectivos, em base populacional.

Há que se destacar, por último, que, em estudos transversais, os efeitos de coorte e de período devem ser considerados, principalmente quando informações agregadas estão disponíveis, sendo que, para o desfecho em questão, as bases de dados existentes no Brasil, até o momento, não contemplam o registro sistemático de morbidade por doenças crônicas em longas séries históricas e, menos ainda, o respectivo registro da prevalência de potenciais fatores de risco para estas doenças, entre os quais se inclui o registro sistemático de prevalência de alcoolismo e seus níveis de dependência.

A identificação da prevalência de dependência de álcool, ao lado da identificação de grupos vulneráveis a este desfecho no Município de Ribeirão Preto, constitui-se em uma ferramenta essencial para o estabelecimento de ações de vigilância que visem medidas efetivas e eficientes de controle, bem como ao planejamento de políticas racionais em Saúde Pública, embasadas na promoção da saúde e na prevenção de comportamentos de risco social, que deverão gerar, por conseguinte, a prevenção de doenças crônicas a eles relacionadas.

\section{Resumo}

O objetivo do estudo foi investigar a prevalencia da dependência de álcool, níveis de gravidade do hábito e identificar fatores associados à dependência. Estudo epidemiológico transversal de base populacional, em amostra de adultos com 30 anos e mais, residentes em Ribeirão Preto, São Paulo, Brasil, em 2006. Com base no questionário AUDIT, prevalências e razões de prevalências (RP) foram calculadas, sendo a variável dependente classificada em duas categorias: (1) escores de 0-7 e (2) escores de 8-40. A prevalência de dependência foi de 20,18\%, sendo, respectivamente, $43,47 \%$ e 10,18\% para os sexos masculino e feminino. O elenco de fatores associados à dependência foi: sexo, idade, nível educacional, estado marital, condição de trabalho, hábito de fumar, filiação religiosa e autopercepção do estado de saúde. A prevalência de dependência foi elevada, principalmente entre os homens. A identificação de fatores associados pode permitir a adoção de estratégias de promoção e prevenção, considerando-se os agravos decorrentes da dependência do álcool.

Alcoolismo; Estudos Transversais; Questionários; Prevalência 


\section{Colaboradores}

S. A. Moraes participou da concepção e planejamento do estudo, da análise e interpretação dos resultados, da revisão crítica de conteúdo e aprovação da versão final do manuscrito. I. C. M. Freitas participou da concepção e planejamento do estudo, da análise e interpretação dos resultados e da elaboração de todas as versões do manuscrito.

\section{Referências}

1. World Health Organization. WHO Expert Committee on problems related to alcohol consumption. $2^{\text {nd }}$ Ed. Geneva: World Health Organization; 2007. (Technical Report Series, 944).

2. Grucza RA, Bucholz KK, Rice JP, Bierut LJ. Secular trends in the lifetime prevalence of alcohol dependence in the United States: a re-evaluation. Alcohol Clin Exp Res 2008; 32:763-70.

3. Giesbrecht N, Ialomiteanu A, Anglin L. Drinking patterns and perspectives on alcohol policy; results from two Ontario surveys. Alcohol Alcohol 2005; 40:132-9.

4. Rehm J, Monteiro M. Alcohol consumption and burden of disease in the Americas: implications for alcohol policy. Rev Panam Salud Pública 2005; 18:241-8.

5. Moraes E, Campos GM, Figlie NB, Laranjeira RR, Ferraz MB. Conceitos introdutórios de economia da saúde e o impacto social do abuso de álcool. Rev Bras Psiquiatr 2006; 28:321-5.

6. Almeida-Filho N, Lessa I, Magalhães L, Araújo MJ, Aquino E, Kawachi I, et al. Alcohol drinking patterns by gender, ethnicity, and social class in Bahia, Brazil. Rev Saúde Pública 2004; 38:45-54.

7. Galduróz JCF, Noto AR, Nappo SA, Carlini ELA. First household survey on drug abuse in São Paulo, Brazil, 1999: principal findings. São Paulo Med J 2003; 12:231-7.
8. Bastos FI, Beroni N, Hacker MA. Consumo de álcool e drogas: principais achados de pesquisa de âmbito nacional, Brasil 2005. Rev Saúde Pública 2008; 42 Suppl 1:109-17.

9. Barros MBA, Botega NJ, Dalgalarrondo P, MarinLeón L, Oliveira HB. Prevalence of alcohol abuse and associated factors in a population-based study. Rev Saúde Pública 2007; 41:502-9.

10. Moraes SA, Freitas ICM, Gimeno SGA, Mondini L. Prevalência de diabetes mellitus e identificação de fatores associados em adultos residentes em área urbana de Ribeirão Preto, São Paulo, Brasil, 2006: Projeto OBEDIARP. Cad Saúde Pública 2010; 26:929-41.

11. Silva NN. Amostragem probabilística: um curso introdutório. São Paulo: Edusp; 2001.

12. Levy PS, Lemeshow S. Sampling of population: methods and applications. $4^{\text {th }}$ Ed. Hoboken: John Wiley \& Sons; 2008.

13. Babor TF, Higgins-Biddle JC, Saunders JB, Monteiro MG. AUDIT: The Alcohol Use Disorders Identification test. Guidelines for use in primary health care. Geneva: Department of Mental Health and Substance Dependence, World Health Organization; 2001.

14. Lima CT, Freire ACC, Silva APB, Teixeira RM, Farrel $\mathrm{M}$, Prince M. Concurrent and construct validity of the AUDIT in an urban Brazilian sample. Alcohol Alcohol 2005; 40:584-9. 
15. Kypri K, McGee R, Saunders JB, Langley JD, Dean JI. Interpretation of items in the AUDIT Questionnaire. Alcohol Alcohol 2002; 37:465-7.

16. Barros AJ, Hirakata VN. Alternatives for logistic regression in cross-sectional studies: an empirical comparison of models that directly estimate the prevalence ratio. BMC Med Res Methodol 2003; 3:21.

17. Kleinbaum DG, Kupper LL, Muller KE, Nizam A. Applied regression analysis and other multivariable methods. 3rd Ed. Pacific Grove: Duxbury Press; 1998.

18. Selin KH. Alcohol Use Disorder Identification Test (AUDIT): what does it screen? Performance of the AUDIT against four different criteria in a Swedish Population Sample. Subst Use Misuse 2006; 41:1881-99.

19. Dolman JM, Hawkes ND. Combining the audit questionnaire and biochemical markers to assess alcohol use and risk of alcohol withdrawal in medical inpatients. Alcohol Alcohol 2005; 40:515-9.

20. Donovan DM, Kivlahan DR, Doyle SR, Longabaugh R, Greenfield SF. Concurrent validity of the Alcohol Use Disorders Identification Test (AUDIT) and AUDIT zones in defining levels of severity among out-patients with alcohol dependence in the COMBINE study. Addiction 2006; 101:1696-704.

21. Costa JSD, Silveira MF, Gazalle FK, Oliveira SS, Hallal PC, Menezes AMB, et al. Consumo abusivo de álcool e fatores associados: estudo de base populacional. Rev Saúde Pública 2004; 38:284-91.
22. Silveira CM, Wang Y-P, Andrade AG, Andrade LH. Heavy episodic drinking in the São Paulo epidemiologic catchment area study in Brazil: gender and sociodemographic correlates. J Stud Alcohol Drugs 2007; 68:18-27.

23. Mendoza-Sassi RA, Béria JU. Prevalence of alcohol use disorders and associated factors: a populationbased study using AUDIT in southern Brazil. Adicction 2003; 98:799-804.

24. Laranjeira R, Pinsky I, Sanchez M, Zaleski M, Caetano R. Alcohol use patterns among Brazilian adults. Rev Bras Psiquiatr 2010; 32:231-41.

25. Lim W-Y, Fong CW, Chan JML, Heng D, Bhalla V, Chew SK. Trends in alcohol consumption in Singapore 1992-2004. Alcohol Alcohol 2007; 42:354-61.

26. Bau CHD. Estado atual e perspectivas da genética e epidemiologia do alcoolismo. Ciênc Saúde Coletiva 2002; 7:183-90.

27. Dawson DA, Stinson FS, Chou P, Grant BF. Threeyear changes in adult risk drinking behavior in relation to the course of alcohol-use disorders. J Stud Alcohol Drugs 2008; 69:866-77.

28. Peluso ETP, Blay SL. Public perception of alcohol dependence. Rev Bras Psiquiatr 2008; 30:19-24.

Recebido em 18/Jul/2010

Versão final reapresentada $07 / J u n / 2011$

Aprovado em 29/Jun/2011 